\title{
Impact of dexmedetomidine on hemodynamics in rabbits $^{1}$
}

Jianjun Ren', Changfa Li", Shuxian Ma"', Jiangli Wu"', Yanjie Yang"'I

'Professor, Department of Anesthesiology, the Second Hospital of Hebei Medical University, Shijiazhuang, China. Conception, design, intellectual and scientific content of the study; manuscript writing; critical revision; final approval the manuscript.

"MD, Department of Anesthesiology, Maternity and Child Health Care of Zaozhuang, China. Acquisition and analysis of data, manuscript writing.

"'MD, Department of Anesthesiology, the Second Hospital of Hebei Medical University, Shijiazhuang, China. Acquisition and analysis of data.

\section{Abstract}

Purpose: To evaluate the effects of single intravenous administration of Dexmedetomidine (DEX) on hemodynamics in rabbits.

Methods: A total of $32 \mathrm{New}$ Zealand white rabbits were randomly divided into the control group (Group C), Group D1 (2.75 $\mu \mathrm{g} / \mathrm{kg})$, Group D2 $(5.5 \mu \mathrm{g} / \mathrm{kg})$, and Group D3 $(8.25 \mu \mathrm{g} / \mathrm{kg})$ to compare systolic blood pressure (SBP), diastolic blood pressure (DBP), heart rate (HR), central venous pressure (CVP), left ventricular systolic pressure (LVSP), left ventricular endstage diastolic pressure (LVEDP), left ventricular developmental pressure (LVDP), $+d p / d t m a x$, -dp/dtmax, and t-dp/dtmax at different time points.

Results: The levels of SBP, DBP, HR, LVSP, and LVEDP in Group D1, D2, and D3 were lower than that of Group C from T1 to T5 $(P<0.05)$, but there was no significant difference at T6 and T7 $(P>0.05)$. Compared with T0, the levels of SBP, DBP, HR, LVSP, LVEDP, and left arterial pressure (LAP) from T1 to T7 were decreased $(P<0.05)$, but there was no significant difference in the other indexes $(P>0.05)$.

Conclusion: Dexmedetomidine can decrease blood pressure and heart rate in rabbits in a dose-dependent manner, but there is no effect on the myocardial systolic and diastolic function.

Key words: Dexmedetomidine. Hemodynamics. Heart Function Tests. Rabbits. 


\section{Introduction}

Dexmedetomidine (DEX) is a specific $\alpha 2$ receptor agonist with sedative, analgesic, and anxiolytic effects. Its selectivity toward $\alpha 2$ receptors is higher than Clonidine (the selection ratio is 1.620:1, which is 8 times that of Clonidine), and it is considered to be a complete agonist of $\alpha 2$ receptors ${ }^{1}$. It is clinically used as an adjuvant drug for intensive care unit (ICU) sedation during mechanical ventilation, intraoperative sedation, and anesthesia. The impact of DEX on the cardiovascular system mainly manifests as negative chronotropic and inotropic effects, namely reduction of the heart rate and blood pressure. Studies have considered that these effects come from DEX's inhibition of the central nervous system and the sympathetic nervous system ${ }^{2}$. There are many reports on the effects of DEX on hemodynamics. Because the study subjects in those reports were primarily patients with surgeries, interference factors such as surgery, anesthesia, or complications may exist ${ }^{3-5}$, together with the differences in the administration modes (sustained infusion after loading dose or avoiding the loading dose), it's difficult to accurately reflect the effects of DEX on hemodynamics ${ }^{6,7}$. Although certain studies have observed the effects of DEX on volunteers' hemodynamics and cardiac function ${ }^{8-10}$, the observation indexes were all derived from indirect assays, and some difficulties exist in determining hemodynamic parameters directly ${ }^{11,12}$.

In this study, rabbits were used as experimental subjects with which to directly measure the hemodynamic parameters and ventricular function indexes after a single administration of DEX with different doses, with the goal of providing references for the clinical application of DEX.

\section{Methods}

The animal use protocol has been reviewed and approved by the Institutional Animal Care and Use Committee (IACUC) of the Second Hospital of Hebei Medical University.

A total of 32 healthy male New Zealand white rabbits, weighing $2.3-2.6 \mathrm{~kg}$, clean grade, were provided by the Experimental Animal Center of Academy of Agricultural Sciences. All the animals were given standard feed for 1-week adaptive feeding with free access to drinking water. The breeding room was kept quiet with the temperature at $26^{\sim} 28^{\circ} \mathrm{C}$ and the relative humidity at $55 \sim 57 \%$. All the rabbits were fasted $8 \mathrm{~h}$ before the experiment, although they still had free access to drinking water. This study was carried out in strict accordance with the recommendations in the Guide for the Care and Use of Laboratory Animals of the National Institutes of Health.

\section{Preparation of experimental animals}

Each rabbit was anesthetized via injection of $0.7 \%$ Pentobarbital sodium $6 \mathrm{ml} / \mathrm{kg}$ into the ear vein and then fixed onto the animal operating table after tested for unconscious. Then, a tracheotomy was performed, followed by intubation for oxygen supply, and the left femoral artery was located and intubated on each rabbit to monitor the systolic blood pressure (SBP), diastolic blood pressure (DBP), and heart rate (HR). The four limbs were connected to needle electrodes for monitoring with an electrocardiogram (ECG). The right internal jugular vein was also isolated and intubated to measure central venous pressure (CVP), and the left common carotid artery was isolated and intubated with a left atrial pressure measuring tube that was approximately $4 \sim 5$ $\mathrm{cm}$. The atrial pressure measurement tube 
was connected to a RM6240 multi-channel physiological signal detector through one YP100 pressure transducer (parameter setting: data acquisition frequency $800 \mathrm{HZ}$, scanning speed $250 \mathrm{~ms} /$ div, calibration sensitivity 90 $\mathrm{mmHg}$, time constant: $\mathrm{DC}$, and filter frequency $30 \mathrm{HZ})$, and when the monitor display and the RM6240 detector showed the ventricular pressure waveform, it was confirmed that the catheter was inserted into the left ventricle. A thoracotomy was then performed of the rabbit, cutting open and exposing the pericardium, and the left auricle was intubated to measure the left arterial pressure (LAP). After all the operations were completed and the rabbit was kept stable for $30 \mathrm{~min}$, all the parameters were recorded as the baseline values.

\section{Grouping}

All the rabbits were randomly divided after surgery into Group C, Group D1, Group D2, and Group D3, with 8 rabbits in each group. A total of $200 \mu \mathrm{g}$ of DEX (Jiangsu Xinchen Co, batch number: 09081232) was diluted with 50 $\mathrm{ml}$ of saline for future use. Group D1 was given $2.75 \mu \mathrm{g} / \mathrm{kg}$ DEX, Group D2 was given DEX 5.5 $\mu \mathrm{g} / \mathrm{kg}$, and Group D3 group was given DEX 8.25 $\mu \mathrm{g} / \mathrm{kg}$, Group C was given the corresponding dose of saline. All administration was via the ear vein using an intravenous infusion pump and was completed within $20 \mathrm{~min}$.

Hemodynamics and cardiac functional indexes

The values of SBP, DBP, HR, left ventricular systolic pressure (LVSP), left ventricular end-stage diastolic pressure (LVEDP), LAP, and CVP at different time points (stable for $30 \mathrm{~min}$ (TO), $1 \mathrm{~min}$ after medication (T1), 5 min after medication (T2), 10 min after medication (T3), 20 min after medication (T4), $30 \mathrm{~min}$ after medication (T5), $60 \mathrm{~min}$ after medication (T6), 120 min after medication (T7)) were taken for each rabbit, and then the left ventricular developmental pressure (LVDP) was calculated (LVDP = LVVD - LVEDP); the RM6240 detector was used to calculate the $+d p / d$ tmax, -Dp/dtmax, and t-dp/dtmax at different time points.

\section{Statistical analysis}

SPSS 8.0 statistical software was used for the analysis; the normally distributed measurement data were expressed as $\bar{x} \pm \mathrm{s}$; the measurement data were analyzed with a repeated measure analysis of variance (ANOVA), and $\mathrm{P}<0.05$ was considered statistically significance.

\section{- Results}

Dose-dependent impact of DEX on hemodynamics

There was no significant difference in all the data at T0 among the groups $(P>0.05)$. Compared with group $C$, there was no significant difference in LVDP, LAP, CVP (Table 1), +dp/ dtmax, -dp/dtmax, and t-dp/dtmax (Table 2) among Group D1, D2, and D3 at all time points $(P>0.05)$. The levels of SBP, DBP, HR (Table 3), LVSP, and LVEDP (Table 1 ) were decreased at T1 in a dose-dependent manner $(P<0.05)$, and the intergroup comparison exhibited statistical significance $(P<0.05)$. The indexes in all the groups returned to baseline values at T6 and T7, and the intergroup comparison exhibited no statistical significance $(P>0.05)$. 
Table 1 - The effects of DEX on LVSP/LVEDP/LVDP and CVP/LAP in rabbits $(n=8, \bar{x} \pm s)$.

\begin{tabular}{|c|c|c|c|c|c|c|c|c|c|}
\hline & $\begin{array}{l}\text { 을 } \\
\text { ํํㄴ }\end{array}$ & TO & T1 & T2 & T3 & T4 & T5 & T6 & $\mathrm{T7}$ \\
\hline \multirow{4}{*}{ 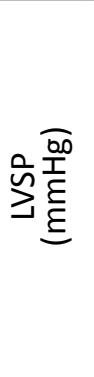 } & C & $\begin{array}{c}158.15 \pm \\
9.47\end{array}$ & $\begin{array}{c}153.41 \pm \\
6.62\end{array}$ & $\begin{array}{c}154.09 \pm \\
6.33\end{array}$ & $\begin{array}{c}158.76 \pm \\
12.14\end{array}$ & $\begin{array}{c}151.94 \pm \\
6.64\end{array}$ & $\begin{array}{c}150.41 \pm \\
5.94\end{array}$ & $\begin{array}{c}154.23 \pm \\
7.41\end{array}$ & $\begin{array}{c}153.10 \pm \\
6.49\end{array}$ \\
\hline & $\mathrm{D} 1$ & $\begin{array}{c}159.41 \pm \\
10.34\end{array}$ & $\begin{array}{c}152.38 \pm \\
10.35\end{array}$ & $\begin{array}{c}146.26 \pm \\
12.08\end{array}$ & $\begin{array}{c}141.40 \pm \\
11.76\end{array}$ & $\begin{array}{c}133.08 \pm \\
13.26 \#\end{array}$ & $\begin{array}{c}140.16 \pm \\
13.99\end{array}$ & $\begin{array}{c}155.64 \pm \\
6.85\end{array}$ & $\begin{array}{c}162.78 \pm \\
10.59\end{array}$ \\
\hline & D2 & $\begin{array}{c}161.77 \pm \\
12.09\end{array}$ & $\begin{array}{c}151.16 \pm \\
12.43\end{array}$ & $\begin{array}{c}143.25 \pm \\
11.59^{\star}\end{array}$ & $\begin{array}{l}126.34 \pm \\
19.00^{\# \star}\end{array}$ & $\begin{array}{l}111.81 \pm \\
20.20^{\# \star}\end{array}$ & $\begin{array}{c}122.43 \pm \\
27.52^{\#}\end{array}$ & $\begin{array}{c}152.33 \pm \\
14.71\end{array}$ & $\begin{array}{c}163.73 \pm \\
16.83\end{array}$ \\
\hline & D3 & $\begin{array}{c}157.01 \pm \\
13.85\end{array}$ & $\begin{array}{c}143.75 \pm \\
15.43\end{array}$ & $\begin{array}{l}127.36 \pm \\
17.01^{\# \star \Delta}\end{array}$ & $\begin{array}{l}105.46 \pm \\
17.28^{\# \star \Delta}\end{array}$ & $\begin{array}{c}90.55 \pm \\
12.07^{\# \star \Delta}\end{array}$ & $\begin{array}{l}97.26 \pm \\
8.98^{\# \star \Delta}\end{array}$ & $\begin{array}{c}140.05 \pm \\
17.07\end{array}$ & $\begin{array}{c}150.31 \pm \\
14.31\end{array}$ \\
\hline \multirow{4}{*}{ 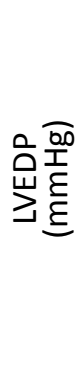 } & C & $\begin{array}{c}129.12 \pm \\
10.15\end{array}$ & $\begin{array}{c}126.13 \pm \\
11.42\end{array}$ & $\begin{array}{c}124.71 \pm \\
9.11\end{array}$ & $\begin{array}{c}136.97 \pm \\
8.65\end{array}$ & $\begin{array}{c}123.71 \pm \\
7.12\end{array}$ & $\begin{array}{c}135.81 \pm \\
8.78\end{array}$ & $\begin{array}{l}125 \pm \\
10.02\end{array}$ & $\begin{array}{c}130.21 \pm \\
10.86\end{array}$ \\
\hline & D1 & $\begin{array}{c}125.12 \pm \\
10.12\end{array}$ & $\begin{array}{c}121.12 \pm \\
8.83\end{array}$ & $\begin{array}{c}113.91 \pm \\
8.51^{\# \star}\end{array}$ & $\begin{array}{c}112.47 \pm \\
8.17^{\# \star}\end{array}$ & $\begin{array}{c}105.43 \pm \\
7.71^{\# \star}\end{array}$ & $\begin{array}{l}109.88 \pm \\
10.58^{\# \star}\end{array}$ & $\begin{array}{c}123.77 \pm \\
4.73\end{array}$ & $\begin{array}{c}131.14 \pm \\
10.80\end{array}$ \\
\hline & D2 & $\begin{array}{c}123.54 \pm \\
12.94\end{array}$ & $\begin{array}{l}116.50 \pm \\
12.95^{\# \star}\end{array}$ & $\begin{array}{l}117.96 \pm \\
12.71^{\# \star}\end{array}$ & $\begin{array}{l}104.16 \pm \\
14.85^{f^{*} \star}\end{array}$ & $\begin{array}{c}84.29 \pm \\
15.09^{\# * \star}\end{array}$ & $\begin{array}{c}95.9 \pm \\
21.61 \#^{* \star}\end{array}$ & $\begin{array}{c}126.76 \pm \\
20.56\end{array}$ & $\begin{array}{c}131.73 \pm \\
8.98\end{array}$ \\
\hline & D3 & $\begin{array}{c}125.29 \pm \\
15.64\end{array}$ & $\begin{array}{l}114.02 \pm \\
14.34^{\#^{\star} \star}\end{array}$ & $\begin{array}{c}98.74 \pm \\
15.49^{\# \star \Delta \star}\end{array}$ & $\begin{array}{c}72.82 \pm \\
14.16^{\# \star \Delta \star}\end{array}$ & $\begin{array}{c}64.95 \pm \\
11.77^{\# \star \Delta \star}\end{array}$ & $\begin{array}{l}66.37 \pm \\
6.1^{7 \#^{*} \Delta \star}\end{array}$ & $\begin{array}{c}107.03 \pm \\
15.45^{\#^{\star} \star \Delta}\end{array}$ & $\begin{array}{l}123.09 \pm \\
14.06^{\# \star \Delta}\end{array}$ \\
\hline \multirow{4}{*}{ 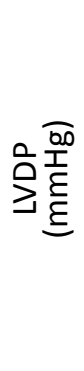 } & C & $\begin{array}{c}30.48 \pm \\
4.31\end{array}$ & $\begin{array}{c}28.925 \pm \\
4.62\end{array}$ & $\begin{array}{c}30.14 \pm \\
4.54\end{array}$ & $\begin{array}{c}27.62 \pm \\
4.26\end{array}$ & $\begin{array}{c}27.02 \pm \\
5.72\end{array}$ & $\begin{array}{c}27.39 \pm \\
3.51\end{array}$ & $\begin{array}{c}30.00 \pm \\
6.09\end{array}$ & $\begin{array}{c}29.42 \pm \\
6.27\end{array}$ \\
\hline & D1 & $\begin{array}{c}34.28 \pm \\
3.13\end{array}$ & $\begin{array}{c}31.256 \pm \\
3.37\end{array}$ & $\begin{array}{c}32.36 \pm \\
4.04\end{array}$ & $\begin{array}{c}28.93 \pm \\
4.16\end{array}$ & $\begin{array}{c}27.64 \pm \\
3.34\end{array}$ & $\begin{array}{c}30.28 \pm \\
4.33\end{array}$ & $\begin{array}{c}31.74 \pm \\
3.86\end{array}$ & $\begin{array}{c}31.66 \pm \\
3.72\end{array}$ \\
\hline & D2 & $\begin{array}{c}38.23 \pm \\
4.97\end{array}$ & $\begin{array}{c}34.66 \pm \\
7.71\end{array}$ & $\begin{array}{c}25.28 \pm \\
3.80\end{array}$ & $\begin{array}{c}22.17 \pm \\
8.08\end{array}$ & $\begin{array}{c}27.54 \pm \\
7.55\end{array}$ & $\begin{array}{l}26.53 \pm \\
8.63^{\star}\end{array}$ & $\begin{array}{l}25.42 \pm \\
8.72^{\star}\end{array}$ & $\begin{array}{l}31.99 \pm \\
7.93\end{array}$ \\
\hline & D3 & $\begin{array}{c}31.71 \pm \\
5.77\end{array}$ & $\begin{array}{c}29.73 \pm \\
5.80\end{array}$ & $\begin{array}{c}28.61 \pm \\
3.78\end{array}$ & $\begin{array}{c}32.64 \pm \\
4.73\end{array}$ & $\begin{array}{l}25.59 \pm \\
7.74\end{array}$ & $\begin{array}{c}30.89 \pm \\
4.40\end{array}$ & $\begin{array}{c}33.02 \pm \\
3.6\end{array}$ & $\begin{array}{c}27.22 \pm \\
6.57\end{array}$ \\
\hline \multirow{4}{*}{ 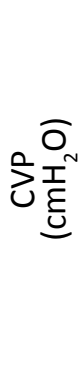 } & C & $\begin{array}{c}3.60 \pm \\
0.48\end{array}$ & $\begin{array}{c}3.80 \pm \\
0.64\end{array}$ & $\begin{array}{c}3.60 \pm \\
0.72\end{array}$ & $\begin{array}{c}3.80 \pm \\
0.64\end{array}$ & $\begin{array}{c}3.40 \pm \\
0.48\end{array}$ & $\begin{array}{c}3.60 \pm \\
0.48\end{array}$ & $\begin{array}{c}3.80 \pm \\
0.64\end{array}$ & $\begin{array}{c}3.40 \pm \\
0.48\end{array}$ \\
\hline & D1 & $\begin{array}{c}3.66 \pm \\
0.66\end{array}$ & $\begin{array}{c}3.50 \pm \\
0.66\end{array}$ & $\begin{array}{c}3.16 \pm \\
0.61\end{array}$ & $\begin{array}{c}3.00 \pm \\
0.33\end{array}$ & $\begin{array}{c}2.83 \pm \\
0.55\end{array}$ & $\begin{array}{c}3.00 \pm \\
0.33\end{array}$ & $\begin{array}{c}3.10 \pm \\
0.50\end{array}$ & $\begin{array}{c}3.00 \pm \\
0.33\end{array}$ \\
\hline & D2 & $\begin{array}{c}4.00 \pm \\
0.80\end{array}$ & $\begin{array}{c}3.60 \pm \\
0.72\end{array}$ & $\begin{array}{c}3.60 \pm \\
0.72\end{array}$ & $\begin{array}{c}3.60 \pm \\
0.72\end{array}$ & $\begin{array}{c}3.60 \pm \\
0.72\end{array}$ & $\begin{array}{c}3.80 \pm \\
0.64\end{array}$ & $\begin{array}{c}3.80 \pm \\
0.64\end{array}$ & $\begin{array}{c}4.00 \pm \\
0.80\end{array}$ \\
\hline & D3 & $\begin{array}{c}3.80 \pm \\
0.55\end{array}$ & $\begin{array}{c}3.60 \pm \\
0.8\end{array}$ & $\begin{array}{c}3.80 \pm \\
0.90\end{array}$ & $\begin{array}{c}4.00 \pm \\
0.33\end{array}$ & $\begin{array}{c}4.00 \pm \\
0.60\end{array}$ & $\begin{array}{c}4.10 \pm \\
0.01\end{array}$ & $\begin{array}{c}5.00 \pm \\
0.50\end{array}$ & $\begin{array}{c}4.30 \pm \\
0.90\end{array}$ \\
\hline \multirow{4}{*}{ } & C & $\begin{array}{c}8.00 \pm \\
0.50\end{array}$ & $\begin{array}{c}7.75 \pm \\
0.37\end{array}$ & $\begin{array}{c}7.50 \pm \\
0.75\end{array}$ & $\begin{array}{c}7.25 \pm \\
0.75\end{array}$ & $\begin{array}{c}7.25 \pm \\
0.37\end{array}$ & $\begin{array}{c}8.00 \pm \\
0.50\end{array}$ & $\begin{array}{c}7.75 \pm \\
0.37\end{array}$ & $\begin{array}{c}7.00 \pm \\
0.50\end{array}$ \\
\hline & D1 & $\begin{array}{c}7.50 \pm \\
0.50\end{array}$ & $\begin{array}{c}7.00 \pm \\
0.50\end{array}$ & $\begin{array}{c}5.75 \pm \\
1.25\end{array}$ & $\begin{array}{c}5.50 \pm \\
1.50\end{array}$ & $\begin{array}{c}7.00 \pm \\
0.66\end{array}$ & $\begin{array}{c}7.00 \pm \\
2.00\end{array}$ & $\begin{array}{l}5.50 \pm \\
0.50 \star\end{array}$ & $\begin{array}{c}6.50 \pm \\
1.50\end{array}$ \\
\hline & D2 & $\begin{array}{c}8.00 \pm \\
0.66\end{array}$ & $\begin{array}{c}7.60 \pm \\
1.77\end{array}$ & $\begin{array}{c}7.30 \pm \\
1.30\end{array}$ & $\begin{array}{l}6.33 \pm \\
1.11^{\star}\end{array}$ & $\begin{array}{l}5.66 \pm \\
0.44^{\star}\end{array}$ & $\begin{array}{l}5.00 \pm \\
0.66^{\star}\end{array}$ & $\begin{array}{c}7.33 \pm \\
1.10\end{array}$ & $\begin{array}{c}7.30 \pm \\
0.40\end{array}$ \\
\hline & D3 & $\begin{array}{c}8.30 \pm \\
0.40 \\
\end{array}$ & $\begin{array}{c}7.60 \pm \\
0.80 \\
\end{array}$ & $\begin{array}{l}7.00 \pm \\
0.40^{\star}\end{array}$ & $\begin{array}{l}5.66 \pm \\
0.40^{\star}\end{array}$ & $\begin{array}{l}6.00 \pm \\
0.60^{\star} \\
\end{array}$ & $\begin{array}{c}7.30 \pm \\
1.10 \\
\end{array}$ & $\begin{array}{c}7.14 \pm \\
0.60 \\
\end{array}$ & $\begin{array}{c}7.00 \pm \\
0.60 \\
\end{array}$ \\
\hline
\end{tabular}

Compared with Group Control ${ }^{*} P<0.05$; Compared with Group D1 ${ }^{*} P<0.05$; Compared with $D 2{ }^{\triangle} P<0.05$; Compared with T0 ${ }^{\star} P<0.05$ 
Table 2 - The effects of DEX on cardiac function in rabbits $(n=8, \bar{x} \pm s)$.

\begin{tabular}{|c|c|c|c|c|c|c|c|c|c|}
\hline & Group & TO & $\mathrm{T} 1$ & T2 & T3 & T4 & T5 & T6 & T7 \\
\hline \multirow{4}{*}{ 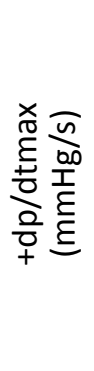 } & $C$ & $\begin{array}{l}1927.51 \\
\pm 417.45\end{array}$ & $\begin{array}{l}1905.26 \\
\pm 463.01\end{array}$ & $\begin{array}{l}1801.71 \\
\pm 278.54\end{array}$ & $\begin{array}{l}1999.72 \\
\pm 404.37\end{array}$ & $\begin{array}{l}1776.24 \\
\pm 264.57\end{array}$ & $\begin{array}{l}1988.51 \\
\pm 433.59\end{array}$ & $\begin{array}{l}1988.51 \\
\pm 433.59\end{array}$ & $\begin{array}{l}1903.76 \\
\pm 341.31\end{array}$ \\
\hline & D1 & $\begin{array}{c}722.02 \\
\pm 456.45\end{array}$ & $\begin{array}{c}11930.78 \\
\pm 712.18\end{array}$ & $\begin{array}{l}1701.99 \\
\pm 367.77\end{array}$ & $\begin{array}{l}1513.10 \\
\pm 537.79\end{array}$ & $\begin{array}{l}1399.61 \\
\pm 465.14\end{array}$ & $\begin{array}{l}1256.75 \\
\pm 287.37\end{array}$ & $\begin{array}{l}1467.86 \\
\pm 265.13\end{array}$ & $\begin{array}{l}1700.57 \\
\pm 472.71\end{array}$ \\
\hline & D2 & $\begin{array}{l}1937.55 \\
\pm 629.31\end{array}$ & $\begin{array}{l}1830.18 \\
\pm 667.32\end{array}$ & $\begin{array}{l}1847.74 \\
\pm 740.81\end{array}$ & $\begin{array}{l}1707.76 \\
\pm 672.24\end{array}$ & $\begin{array}{l}1595.85 \\
\pm 662.65\end{array}$ & $\begin{array}{l}1608.54 \\
\pm 574.51\end{array}$ & $\begin{array}{l}1953.91 \\
\pm 740.20\end{array}$ & $\begin{array}{r}1218.09 \\
\pm 226.59\end{array}$ \\
\hline & D3 & $\begin{array}{l}1927.51 \\
\pm 417.45\end{array}$ & $\begin{array}{l}1861.77 \\
\pm 113.47\end{array}$ & $\begin{array}{c}1495.31 \\
\pm 57.59\end{array}$ & $\begin{array}{c}1572.46 \\
\pm 60.48\end{array}$ & $\begin{array}{c}1426.88 \\
\pm 46.59\end{array}$ & $\begin{array}{c}1469.7 \\
\pm 117.53\end{array}$ & $\begin{array}{l}1611.70 \\
\pm 154.75\end{array}$ & $\begin{array}{c}1498.53 \\
\pm 53.85\end{array}$ \\
\hline \multirow{4}{*}{ 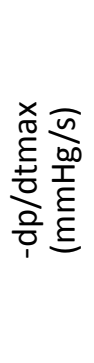 } & C & $\begin{array}{l}1232.63 \\
\pm 160.99\end{array}$ & $\begin{array}{l}1165.69 \\
\pm 156.96\end{array}$ & $\begin{array}{l}1236.52 \\
\pm 101.23\end{array}$ & $\begin{array}{l}1169.68 \\
\pm 161.97\end{array}$ & $\begin{array}{l}1167.23 \\
\pm 123.52\end{array}$ & $\begin{array}{l}1322.04 \\
\pm 209.61\end{array}$ & $\begin{array}{l}1301.72 \\
\pm 142.23\end{array}$ & $\begin{array}{r}1210.93 \\
\pm 130.95\end{array}$ \\
\hline & D1 & $\begin{array}{l}1253.02 \\
\pm 191.26\end{array}$ & $\begin{array}{l}1099.36 \\
\pm 187.90\end{array}$ & $\begin{array}{l}1126.62 \\
\pm 118.78\end{array}$ & $\begin{array}{c}1017.72 \\
\pm 69.88\end{array}$ & $\begin{array}{l}1030.76 \\
\pm 140.03\end{array}$ & $\begin{array}{l}1005.56 \\
\pm 135.66\end{array}$ & $\begin{array}{c}1133.71 \\
\pm 47.81\end{array}$ & $\begin{array}{l}1315.29 \\
\pm 283.50\end{array}$ \\
\hline & D2 & $\begin{array}{l}1205.83 \\
\pm 280.39\end{array}$ & $\begin{array}{l}1366.35 \\
\pm 514.37\end{array}$ & $\begin{array}{l}1243.23 \\
\pm 289.76\end{array}$ & $\begin{array}{l}1202.74 \\
\pm 271.90\end{array}$ & $\begin{array}{l}1084.53 \\
\pm 262.87\end{array}$ & $\begin{array}{l}1078.46 \\
\pm 183.32\end{array}$ & $\begin{array}{l}1278.97 \\
\pm 281.30\end{array}$ & $\begin{array}{c}970.492 \\
\pm 40.15\end{array}$ \\
\hline & D3 & $\begin{array}{l}1222.63 \\
\pm 160.99\end{array}$ & $\begin{array}{l}1256.58 \\
\pm 194.10\end{array}$ & $\begin{array}{l}1214.69 \\
\pm 254.60\end{array}$ & $\begin{array}{c}1110.82 \\
\pm 34.21\end{array}$ & $\begin{array}{l}1186.54 \\
\pm 129.38\end{array}$ & $\begin{array}{l}1054.20 \\
\pm 134.55\end{array}$ & $\begin{array}{l}1180.87 \\
\pm 105.40\end{array}$ & $\begin{array}{c}1021.46 \\
\pm 91.12\end{array}$ \\
\hline \multirow{4}{*}{ 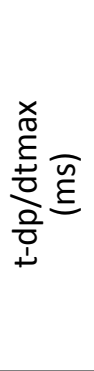 } & $C$ & $\begin{array}{l}17.36 \\
\pm 3.87\end{array}$ & $\begin{array}{l}18.76 \\
\pm 4.34\end{array}$ & $\begin{array}{l}15.60 \\
\pm 2.53\end{array}$ & $\begin{array}{l}16.67 \\
\pm 4.11\end{array}$ & $\begin{array}{l}16.01 \\
\pm 2.48\end{array}$ & $\begin{array}{l}16.41 \\
\pm 2.64\end{array}$ & $\begin{array}{l}15.19 \\
\pm 2.05\end{array}$ & $\begin{array}{l}16.45 \\
\pm 3.04\end{array}$ \\
\hline & D1 & $\begin{array}{l}19.10 \\
\pm 2.98\end{array}$ & $\begin{array}{l}17.99 \\
\pm 4.93\end{array}$ & $\begin{array}{l}19.08 \\
\pm 6.89\end{array}$ & $\begin{array}{l}27.71 \\
\pm 9.45\end{array}$ & $\begin{array}{r}32.00 \\
\pm 17.45\end{array}$ & $\begin{array}{c}32.72 \\
\pm 16.51\end{array}$ & $\begin{array}{l}22.85 \\
\pm 2.18\end{array}$ & $\begin{array}{l}14.64 \\
\pm 2.42\end{array}$ \\
\hline & D2 & $\begin{array}{l}15.84 \\
\pm 3.28\end{array}$ & $\begin{array}{l}15.97 \\
\pm 3.36\end{array}$ & $\begin{array}{l}19.71 \\
\pm 7.84\end{array}$ & $\begin{array}{l}24.48 \\
\pm 7.80\end{array}$ & $\begin{array}{c}30.83 \\
\pm 16.36\end{array}$ & $\begin{array}{l}19.99 \\
\pm 5.36\end{array}$ & $\begin{array}{l}16.31 \\
\pm 3.63\end{array}$ & $\begin{array}{l}14.81 \\
\pm 3.49\end{array}$ \\
\hline & D3 & $\begin{array}{l}20.77 \\
\pm 5.51\end{array}$ & $\begin{array}{l}18.70 \\
\pm 4.00\end{array}$ & $\begin{array}{l}15.15 \\
\pm 2.24\end{array}$ & $\begin{array}{l}22.95 \\
\pm 2.20\end{array}$ & $\begin{array}{l}22.47 \\
\pm 2.33\end{array}$ & $\begin{array}{l}16.55 \\
\pm 3.59\end{array}$ & $\begin{array}{l}15.83 \\
\pm 2.76\end{array}$ & $\begin{array}{l}18.53 \\
\pm 1.53\end{array}$ \\
\hline
\end{tabular}

Note: Compared with Group Control, ${ }^{*} P<0.05$; Compared with Group D1, ${ }^{*} P<0.05$; Compared with D2, $\triangle P<0.05$; Compared with T0, $\star P<0.05$.

Table 3 - The effects of DEX on blood pressure and heart rates in rabbits $(n=8, \bar{x} \pm s)$.

\begin{tabular}{|c|c|c|c|c|c|c|c|c|c|}
\hline & Group & TO & T1 & T2 & T3 & T4 & T5 & T6 & T7 \\
\hline \multirow{4}{*}{ 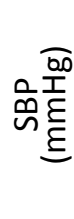 } & C & $134 \pm 11$ & $132 \pm 9$ & $135 \pm 10$ & $131 \pm 13$ & $134 \pm 17$ & $130 \pm 14$ & $129 \pm 10$ & $130 \pm 13$ \\
\hline & D1 & $131 \pm 15$ & $124 \pm 14$ & $119 \pm 13^{\star}$ & $113 \pm 9^{\# \star}$ & $104 \pm 9^{\# \star}$ & $111 \pm 12^{\# \star}$ & $128 \pm 9$ & $135 \pm 15$ \\
\hline & D2 & $135 \pm 18$ & $125 \pm 18^{\star}$ & $118 \pm 15^{\# \star}$ & $104 \pm 21^{\#^{*} \star}$ & $86 \pm 22^{\#^{* \star}}$ & $97 \pm 29^{\#^{*} \star}$ & $124 \pm 16^{\star}$ & $133 \pm 18$ \\
\hline & D3 & $134 \pm 14$ & $121 \pm 15^{\# \star}$ & $104 \pm 21^{\# * \Delta \star}$ & $79 \pm 22^{\# * \Delta \star}$ & $59 \pm 16^{\# * \Delta \star}$ & $70 \pm 16^{\# * \Delta \star}$ & $113 \pm 19^{\# * \Delta \star}$ & $128 \pm 12$ \\
\hline \multirow{4}{*}{ } & C & $89 \pm 6$ & $86 \pm 8$ & $85 \pm 3$ & $87 \pm 5$ & $84 \pm 7$ & $84 \pm 6$ & $85 \pm 4$ & $82 \pm 3$ \\
\hline & D1 & $87 \pm 6$ & $82 \pm 4$ & $81 \pm 5$ & $74 \pm 4^{\star}$ & $68 \pm 6^{\# \star}$ & $73 \pm 5^{\# \star}$ & $84 \pm 3$ & $88 \pm 3$ \\
\hline & D2 & $86 \pm 10$ & $81 \pm 11$ & $76 \pm 9^{\# \star}$ & $66 \pm 9^{\# * \star}$ & $53 \pm 9^{\#^{* \star}}$ & $61 \pm 14^{\#^{*} \star}$ & $81 \pm 6^{\star}$ & $88 \pm 7$ \\
\hline & D3 & $86 \pm 8$ & $77 \pm 9^{\# \star}$ & $65 \pm 11^{\# * \Delta \star}$ & $45 \pm 11^{\#^{*} \Delta \star}$ & $37 \pm 8^{\# *^{*} \Delta}$ & $41 \pm 7^{\# * \Delta \star}$ & $68 \pm 15^{\#^{*} \Delta \star}$ & $79 \pm 9$ \\
\hline \multirow{4}{*}{ 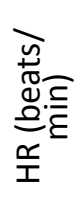 } & C & $258 \pm 15$ & $254 \pm 26$ & $252 \pm 19$ & $258 \pm 23$ & $253 \pm 22$ & $258 \pm 29$ & $256 \pm 19$ & $251 \pm 23$ \\
\hline & D1 & $253 \pm 26$ & $232 \pm 24^{\#}$ & $224 \pm 25^{\# \star}$ & $217 \pm 27^{\# \star}$ & $203 \pm 24^{\# \star}$ & $207 \pm 28^{\# \star}$ & $228 \pm 27^{\# \star}$ & $251 \pm 21$ \\
\hline & D2 & $261 \pm 18$ & $247 \pm 24^{\star}$ & $239 \pm 25^{* \star}$ & $217 \pm 33^{* \star}$ & $189 \pm 33^{* \star}$ & $201 \pm 44^{* \star}$ & $238 \pm 30 * \star$ & $254 \pm 32$ \\
\hline & D3 & $257 \pm 37$ & $241 \pm 46^{\# \star}$ & $225 \pm 41^{\# *^{*} \Delta \star}$ & $188 \pm 32^{\# * \Delta \star}$ & $155 \pm 26^{\# * \Delta \star}$ & $161 \pm 28^{\# * \Delta \star}$ & $231 \pm 49^{\# * \Delta \star}$ & $245 \pm 46$ \\
\hline
\end{tabular}

Compared with Group Control ${ }^{*} P<0.05$; Compared with Group D1 ${ }^{*} P<0.05$; Compared with $\mathrm{D} 2{ }^{\triangle} P<0.05$; Compared with $\mathrm{T0}{ }^{\star} P<0.05$ 
Time-dependent impact of DEX on hemodynamics

There was no significant difference in the intragroup comparison of all the indexes in Group C ( $P>0.05)$. There was no significant difference in the intragroup comparison of CVP (Table 1), +dp/dtmax, -dp/dtmax, and t-dp/dtmax (Table 2) in Group D1, D2, and D3 $(P>0.05)$, but SBP, DBP, HR (Table 3), LVSP, LVEDP, and LAP (Table 1) all decreased to the lowest levels at T1, and those in Group D1 and D2 decreased to the lowest levels at T4, and they returned to normal at T6 and T7 $(P>0.05)$. The indexes in Group D3 were decreased significantly at $\mathrm{T} 2$ and were at their lowest levels at T4, returning to normal at T7 $(P>0.05)$.

\section{Detection of plasma concentration of DEX}

There were statistically significant differences in the intergroup comparison of the plasma concentration of DEX among Group D1, D2, and D3 at T2, T3, T4, and T5 $(P<0.05)$, whereas there was no significant difference at T6 and T7 $(P>0.05)$. The plasma concentrations of DEX in the three groups reached its peak at T1 $(0.72 \pm 0.35 \mathrm{ng} / \mathrm{ml}, 1.70 \pm 1.23 \mathrm{ng} / \mathrm{ml}$, and 3.81 $\pm 1.36 \mathrm{ng} / \mathrm{ml}$, respectively) and then gradually decreased. There were statistically significant $(P<0.05$, Table 4$)$ differences in the intragroup comparison of the plasma concentration of DEX for each group.

Table 4 - The plasma concentration of Dexmedetomidine on every time point $(n=8, \bar{x} \pm s)$.

\begin{tabular}{cccccccc}
\hline Group & T1 & T2 & T3 & T4 & T5 & T6 & T7 \\
\hline D1 & $0.11 \pm 0.12$ & $0.46 \pm 0.20^{\Delta}$ & $0.72 \pm 0.35^{\Delta}$ & $0.66 \pm 0.36^{\Delta}$ & $0.26 \pm 0.09^{\Delta}$ & $0.10 \pm 0.03$ & $0.03 \pm 0.01^{\Delta}$ \\
D2 & $0.29 \pm 0.05^{\#}$ & $1.20 \pm 0.79^{\# \Delta}$ & $1.70 \pm 1.23^{\# \Delta}$ & $1.21 \pm 0.69^{\# \Delta}$ & $0.41 \pm 0.21^{\# \Delta}$ & $0.15 \pm 0.08$ & $0.10 \pm 0.01$ \\
D3 & $1.02 \pm 0.10^{\# *}$ & $2.16 \pm 0.68^{\# * \Delta}$ & $3.81 \pm 1.36^{\#^{* \Delta}}$ & $2.06 \pm 0.16^{\# * \Delta}$ & $0.57 \pm 0.08^{\# \Delta}$ & $0.18 \pm 0.02^{\Delta}$ & $0.09 \pm 0.01$ \\
\hline
\end{tabular}

Compared with Group D1 ${ }^{\#} P<0.05$; Compared with D2 ${ }^{*} P<0.05$; Compared with T0 ${ }^{\triangle} P<0.05$; Compared with T1 ${ }^{\star} P<0.05$.

\section{- Discussion}

Dosage selection of DEX and changes in plasma concentration

The recommended clinical administration mode of DEX by FDA is a loading dose $1 \mu \mathrm{g} / \mathrm{kg}$, infusion time $>10 \mathrm{~min}$, followed by a maintenance dose $0.2-0.7 \mu \mathrm{g} / \mathrm{kg} / \mathrm{h}$ with the medication time of $24 \mathrm{~h}^{13}$. In this study, we administrated a single dose of $2.75 \mu \mathrm{g} / \mathrm{kg}$ (clinically equivalent dose) ${ }^{14}$, as well as double and triple doses of DEX within $20 \mathrm{~min}$, to observe the dose- and time-dependent effects of DEX on hemodynamics in rabbits. The effects of DEX on the cardiovascular system are related to the plasma drug concentration ${ }^{15,16}$.
In this study, the highest concentration of DEX in the three groups appeared at $\mathrm{T} 1$, which were $0.72 \mathrm{ng} / \mathrm{ml}, 1.70 \mathrm{ng} / \mathrm{ml}$, and $3.81 \mathrm{ng} / \mathrm{ml}$ in the three groups, respectively, and the decreasing trend began at T2 with $0.03 \mathrm{ng} / \mathrm{ml}, 0.10 \mathrm{ng} /$ $\mathrm{ml}$, and $0.09 \mathrm{ng} / \mathrm{ml}$ at $\mathrm{T} 7$, respectively. The hemodynamic indexes at different time points changed accordingly.

\section{Impact of DEX on BP and $H R$}

The mechanisms of DEX on the cardiovascular system are much more complex. First, it can act on the central and peripheral nervous system and produce a sympathetic block. However, it acts on the vascular $\alpha 2$ receptors and produces vasoconstriction or a relaxation effect, with the clinical 
manifestations appearing as HR/BP reduction, or diphasic changes of BP. DEX acts on the vascular smooth muscle $\alpha 2$ receptors and produces a vasoconstriction effect, whereas it produces a vasodilation effect when it acts on the vascular endothelial cell $\alpha 2$ receptors ${ }^{17,18}$, thus leading to an increase or decrease of BP. The results of this study show that administrating a single clinically equivalent dose of DEX $(2.75 \mu \mathrm{g} / \mathrm{kg})$ can slightly reduce HR and $\mathrm{BP}$, which were significantly reduced when at the dose of $5.5 \mu \mathrm{g} / \mathrm{kg}$ and $8.25 \mu \mathrm{g} / \mathrm{kg}$. The HR and $B P$ reached their lowest at $20 \mathrm{~min}$ in a dosedependent manner, consistent with previous reports ${ }^{16}$. After medication stopped, HR and BP exhibited an increasing trend with the gradual decline of the plasma drug concentration and return to normal at $60 \mathrm{~min}$, indicating that the hemodynamic effects of DEX are related to its plasma concentration.

\section{Impact of DEX on left ventricular systolic} function

LVSP, +dp/dtmax, and t-dp/dtmax are the common indexes for reflecting myocardial contractility. LVSP is commonly used for detecting CSP in the isovolumic contraction period and is generally positively associated with myocardial contractility. In this study, no significant change was found in $+d p /$ dtmax and t-dp/dtmax at each time point in group D1, D2s, and D3 when compared with that of Group C, suggesting that under the present study conditions, the effects of DEX on myocardial contractility is not significantly related to dose. However, in Group D1, D2, and D3, LVSP decreased with the increase of the plasma concentration, and was gradually restored to the baseline level with the decrease of the DEX concentration; however, the values of $\mathrm{t}$-dp/dtmax and $+\mathrm{dp} / \mathrm{dtmax}$ at different time points in each group showed no significant change, leading to an inconsistency in the indexes of cardiac systolic function. For example, LVSP exhibited an inconsistent trend with $+d p / d t m a x$ and t-dp/dtmax. LVSP represents the ventricular pressure changes in the isovolumic contraction period, and can be greatly affected by the pre- and post-load, as well as HR. In this study, while detecting LVEDP, LAP was also detected, and decreased between $20 \mathrm{~min}$ to $30 \mathrm{~min}$ of medication in the three groups, suggesting that the application of DEX decreased the cardiac pre-load, and the simultaneously changed HR also affecting LVSP. Thus, LVSP could not reflect the actual changes in myocardial contractility. +dp/dtmax is one index in the isovolumic contraction period that can indirectly reflect the shortening speed of myocardial contraction components. Post-load does not affect $+d p / d t m a x$ significantly, but $+d p / d t m a x$ is very sensitive to the pre-load changes and decreases with the decrease of the pre-load. The results of this study show that DEX reduces the pre-load but does not correspondingly reduce $+d p / d t m a x . t-d p /$ dtmax does not change depending on the preload, and can also reliably reflect the myocardial contractility when HR changes; thus, it can more reliably reflect the myocardial systolic function than LVSP and $+\mathrm{dp} / \mathrm{dtmax}^{19}$. LVDP can reflect the myocardial contractility. Although LVSP and LVEDP were decreased after administration of DEX, LVDP did not show significant changes, suggesting that DEX had no significant effects on the cardiac systolic function.

The changes of dp/dtmax, t-dp/dtmax, and LVDP in this study show that DEX has no effect on the ventricular systolic function in rabbits under experimental conditions.

\section{Impact of DEX on left ventricular diastolic} function

The results did not show that DEX had an inhibitory effect on the myocardial 
diastolic function. - dp/dtmax primarily reflects the diastolic function during the ventricular diastolic process and is a sensitive index that can reflect the diastolic parameters and diastolic functional changes in the early myocardial relaxation period. The reduction of $-\mathrm{dp} / \mathrm{dtmax}$ represents the decrease of myocardial diastolic function. The results of this study revealed no significant intra- and intergroup difference in -dp/dtmax among group D1, D2, and D3, consistent with the changing trend of $+\mathrm{dp} /$ dtmax.

Previous studies have suggested that DEX inhibits cardiac function, thus resulting in the reduction of $B P, H R$, cardiac output, and stroke volume ${ }^{10,16}$, which was inconsistent with our results. The reasons may be because the above studies only observed indexes in the cardiac ejection period, but did not carefully observe the cardiac preload and left ventricular systolic/diastolic function indexes.

Comprehensively assessing the left ventricular function requires monitoring the indexes in the systolic, active diastolic, and passive diastolic periods. Ideal indexes for evaluating the contraction function should have the following characteristics: sensitive to inotropic factors, insensitive to pre- and postload and HR, and independent of cardiac size. These indexes include the pressure-volume indexes (LVSP or $+d p / d t m a x)$ and stress response indexes (cardiac output or ejection fraction). Ventricular diastole is a process with multiple factors, which is active, energy-requiring, and closely related to the intra-cardiomyocyte calcium transport ${ }^{20,21}$. Comprehensive diastolic function evaluation indexes should include the active diastolic indexes (time constant T) and passive diastolic indexes (diastolic pressurevolume relationship).

This study did not monitor nor discuss the effects of napental anesthesia on the cardiac functions primarily because of the following two reasons.
First, the intra-experimental anesthesia of the animals used $0.7 \%$ sodium pentobarbital $(6 \mathrm{ml} / \mathrm{kg})$, and the anesthesia maintenance time was approximately $2 \mathrm{~h}$, which was consistent with reference ${ }^{22}$. In this study, before applying dexmedetomidine, operations were performed under local anesthesia with $0.5 \%$ bupivacaine, including: 1 . Tracheostomy, intubation, and oxygenation; 2 . Dissection and exposure of the carotid artery, catheterization to the left ventricle for measuring the left ventricular functional indexes; 3. Dissection and exposure of the internal jugular vein, catheterization to the right atrium for measuring the central venous pressure; 4 . Exposure of the femoral artery, catheterization to the arterial catheter for measuring the blood pressure and connecting the monitor; and 5. Opening of the chest to expose the heart, catheterization to the left atrial appendage for measuring the left atrial pressure. The period from intravenous administration of pentobarbital sodium to finishing the above operations was approximately $2 \mathrm{~h}$, and dexmedetomidine was administrated at the time when the effect of pentobarbital sodium on cardiac function had almost disappeared. In addition, no further pentobarbital sodium anesthesia was supplemented to the animals after dexmedetomidine was administrated. Thus, the indexes in the groups with different doses of dexmedetomidine can be regarded as being only affected by dexmedetomidine, and should accurately reflect the effects of dexmedetomidine on the rabbit heart functions.

\section{- Conclusions}

Single administration of different doses of dexmedetomidine can decrease the blood pressure and heart rate in rabbits but has no effect on the left ventricular systolic and diastolic function. Intraoperative changes in 
blood pressure caused by DEX can be achieved by adjusting HR and peripheral vascular resistance.

\section{References}

1. Weerink MAS, Struys MMRF, Hannivoort LN, Barends CRM, Absalom AR, Colin P. Clinical pharmacokinetics and pharmacodynamics of dexmedetomidin. Clin Pharmacokinet. 2017 Aug;56(8):893-913. doi: 10.1007/ s40262-017-0507-7.

2. Devasya A,Sarpangala M.Dexmedetomidine: a review of a newer sedative in dentistry. J Clin Pediatr Dent. 2015 Fall;39(5):401-9. doi: 10.17796/1053-4628-39.5.401.

3. Tobise F, Toyosmima Y, Kawana S. Effect of dexmedetomidine on hemodynamics in pediatric patients following cardiac surgery. Masui. 2007 Apr;56(4):409-13. PMID: 17441447.

4. Callaway CW, Elmer J, Guyette FX, Molyneaux BJ, Anderson KB, Empey PE, Gerstel SJ, Holquist K, Repine MJ, Rittenberger JC. Dexmedetomidine reduces shivering during mild hypothermia in waking subjects. PLoS One. 2015 Aug 3;10(8):e0129709. doi: 10.1371/journal.pone.0129709.

5. Bekker A, Sturaitis $M$, Bloom M, Moric M, Golfinos J, Parker E, Babu R, Pitti A. The effect of dexmedetomidine on perioperative hemodynamics in patients undergoing craniotomy. Anesth Analg. 2008 Oct;107(4):1340-7. doi: 10.1213/ ane.0b013e3181804298.

6. Mukhtar AM, Obayah EM, Hassona AM. The use of dexmedetomidine in pediatric cardiac surgery. Anesth Analg. 2006 Jul;103(1):52-6. doi:10.1213/01.ane.0000217204.92904.76.

7. Ickeringill $M$, Shehabi $Y$, Adamson $H$, Ruettimann $U$. Dexmedetomidine infusion without loading dose in surgical patients requiring mechanical ventilation: haemodynamic effects and efficacy. Anaesth Intensive Care. 2004 Dec;32(6):741-5. PMID: 15648981.

8. Ishibashi C, Hayashida $M$, Sugasawa Y, Yamaguchi K, Tomita N, Kajiyama Y, Inada E. Effects of dexmedetomidine on hemodynamics and respiration in intubated, spontaneously breathing patients after endoscopic submucosal dissection for cervical esophageal or pharyngeal cancer. J Anesth. 2016 Aug;30(4):628-36. doi: 10.1007/s00540-016-2175-4.

9. Frölich MA, Arabshahi A, Katholi C, Prasain J, Barnes S. Hemodynamic characteristics of midazolam, propofol, and dexmedetomidine in healthy volunteers. J Clin Anesth. 2011 May;23(3):218-23. doi: 10.1016/j. jclinane.2010.09.006.

10.Snapir A, Posti J, Kentala E, Koskenvuo J, Sundell J, Tuunanen H, Hakala K, Scheinin H, Knuuti J, Scheinin M. Effects of low and high plasma concentrations of dexmedetomidine on myocardial perfusion and cardiacfunction in healthy male subjects. Anesthesiology. 2006 Nov;105(5):902-10. PMID: 17065883.

11.Basar $H$, Akpinar S, Doganci N, Buyukkocak U, Kaymak C, Sert O, Apan A. The effects of preanesthetic, singledose dexmedetomidine on induction, hemodynamic, and cardiovascular parameters. J Clin Anesth. 2008 Sep;20(6):431-6. doi: 10.1016/j. jclinane.2008.04.007.

12. Yu M, Han C, Jiang X, Wu X, Yu L, Ding Z. Effect and placental transfer of dexmedetomidine during caesarean section under general anaesthesia. Basic Clin Pharmacol Toxicol. 2015 Sep;117(3):204-8. doi: 10.1111/ bcpt.12389.

13.Gerlach AT, Dasta JF. Dexmedetomidine: an updated review. Ann Pharmacother. 2007 Mar;41(3):530-1. doi: 10.1345/aph.1H314.

14.de Pereira Cardoso HD, Fim NC, Marques MA, Marques MA, Mint $H$, de Vasconcelos Machado VM, Solanki DR, Lima RM, de Carvalho AL, Navarro LH, Ganem EM. Clinical and histological effects of the intrathecal administration of a single dose of dexmedetomidine in rabbits. Pain Physician. 2016 Feb;19(2):E319-27. PMID: 26815259.

15.Bhana N, Goa KL, McClellan KJ. Dexmedetomidine. Drugs. 2000;59(2):26370. PMID: 10730549.

16.Ebert TJ, Hall JE, Barney JA, Uhrich TD, Colinco MD. The effects of increasing plasma concentrations of dexmedetomidine in humans. Anesthesiology. 2000 Aug;93(2):382-94. PMID: 10910487.

17. Yabuki A, Higuchi $H$, Yoshitomi T, Tomoyasu Y, Ishii-Maruhama M, Maeda S, Miyawaki T. Locally injected dexmedetomidine induces vasoconstriction via peripheral $\alpha-2 A$ 
adrenoceptor subtype in guinea pigs. Reg Anesth Pain Med. 2014 Mar-Apr;39(2):133-6. doi: 10.1097/AAP.0000000000000048.

18.FigueroaXF, PobleteMI,BoricMP,Mendizábal VE, Adler-Graschinsky E, Huidobro-Toro JP. Clonidine-induced nitric oxide-dependent vasorelaxation mediated by endothelial alpha(2)-adrenoceptor activation. $\mathrm{Br} J$ Pharmacol. 2001 Nov;134(5):957-68. doi: 10.1038/sj.bjp.0704320.

19.Akimoto $\mathrm{T}$, Hashimoto $\mathrm{S}$, Sunada $\mathrm{K}$. Dexmedetomidine $(12.5 \mu \mathrm{g} / \mathrm{mL})$ improves tissue distribution, anesthetic action, and hemodynamic effects of lidocaine after palatal infiltration in rats. Odontology. 2016 Sep;104(3):390-6. doi: 10.1007/s10266015-0221-6.
20.Bettschart-Wolfensberger $\mathrm{R}$, Freeman SL, Bowen IM, Aliabadi FS, Weller R, Huhtinen $\mathrm{M}$, Clarke KW. Cardiopulmonary effects and pharmacokinetics of i.v. dexmedetomidine in ponies. Equine Vet J. 2005 Jan;37(1):60-4. PMID: 15651736.

21.Campbell KS, Sorrell VL. Cell- and molecularlevel mechanisms contributing to diastolic dysfunction in HFpEF. J Appl Physiol (1985). 2015 Nov 15;119(10):1228-32. doi: 10.1152/ japplphysiol.01168.2014.

22.Liu WP, Bai M. Investigating anesthetic concentration of pentobarbital sodium in rabbits. Acta Medicinae Sinic. 2013 Feb;26(1):11-3.

\section{Correspondence:}

Jianjun Ren

Department of Anesthesiology

The Second Hospital of Hebei Medical University

215 Heping West Road in Xinhua District

Shijiazhuang 050000 China

Phone: +8631166002908

cnjianjunren@126.com

Received: Dec 19, 2017

Review: Feb 17, 2018

Accepted: Mar 18, 2018
Conflict of interest: none

Financial source: none
${ }^{1}$ Research performed at Department of Central Laboratory, the Second Hospital of Hebei Medical University, Shijiazhuang, China. 